\title{
sciendo
}

Int. J. of Applied Mechanics and Engineering, 2021, vol.26, No.4, pp.113-127

DOI: 10.2478/ijame-2021-0053

\section{EFFECTS OF THE CONTROL PARAMETERS ON THE STABILITY OF A LAMINAR BOUNDARY LAYER ON A POROUS FLAT PLATE}

\author{
T.F. LIHONOU ${ }^{1}$, A.V. MONWANOU ${ }^{1 *}$, C.H. MIWADINOU ${ }^{1,2}$ and J.B. CHABI OROU ${ }^{1}$ \\ ${ }^{1}$ Laboratoire de Mécanique des Fluides, de la Dynamique Nonlinéaire et de la Modélisation des Systèmes \\ Biologiques (LMFDNMSB), Institut de Mathématiques et de Sciences Physiques(IMSP)/UAC \\ BP: 613 Porto-Novo, BENIN \\ ${ }^{2}$ Ecole Normale Supérieure de Natitingou (ENS), Université Nationale des Sciences, Technologies \\ Ingénierie et Mathématiques (UNSTIM) d'Abomey, BENIN \\ E-mails: movins2008@yahoo.fr, vincent.monwanou@imsp-uac.org
}

\begin{abstract}
This work is devoted to the analysis of the linear temporal stability of a laminar dynamic boundary layer on a horizontal porous plane plate. The basic flow is assumed to be laminar and two-dimensional. The basic flow velocity profiles are obtained by numerically solving the Blasius equation using the Runge-Kutta method. The perturbations of these basic solutions are expressed in the form of three-dimensional Tollmien-Schlichting waves. The formulation of the stability problem leads to the Orr-Sommerfeld equation modified by the permeability parameter (Darcy number) and the small Reynolds number. This equation is given in a general form which can be applied to the Chebyshev domain and the boundary layer domain and solved numerically using the Chebyshev spectral collocation method. The marginal stability diagrams, the critical Reynolds numbers and the eigenvalue spectra are obtained for different values of the parameters which have modified the stability equation. Numerical solutions indicate the importance of the effect of these parameters on the flow stability characteristics.
\end{abstract}

Key words: permeability parameter, small Reynolds number, linear temporal stability, boundary layer, modified Orr-Sommerfeld equation, spectral collocation method.

\section{Introduction}

Research to improve aeronautical performance, reduce fuel consumption and reduce noise emissions is an important aspect within the scientific community interested in the development of aerodynamic vehicles. Thus, flow control and the problem of reducing drag and vortex instabilities are topical themes that are the subject of much research in several laboratories around the world. To this end, various flow control techniques have been used in order to control the flow; they fall into two broad categories: passive control and active control. Passive techniques are generally simple to implement and inexpensive, since they do not require an external input of energy; they mainly use geometric or physical characteristics to act on the flow [1,2]. Active techniques require an external input of energy which must be as low as possible to optimize the energy supplied/energy gain ratio. They use information relating to the flow to adapt its action $[3,4]$, such as for example control by injection or aspiration.

Among the research work carried out with active control on the study of the temporal linear stability of a flow, we can mention among others Ming-Liang et al. [5] who derived the parabolized stability equation (PSE) to study the linear stability of the charged particle flow in the growing Blasius boundary layer. The stability characteristics for various Stokes numbers and particle concentrations were analyzed after solving the equation numerically using the perturbation and finite difference method. Adding fine particles reduces the critical Reynolds number while adding coarse particles improves it. The stabilizing and destabilizing effect of the particles depends monotonically on the concentration of the particles, but not monotonically on the Stokes number.

\footnotetext{
${ }^{*}$ To whom correspondence should be addressed
} 
Seth et al. [6] studied the Hartmann flow of an electrically conductive incompressible viscous fluid in a rotating system in the presence of an inclined magnetic field. Expressions for the shear stress at the channel plates due to the primary and secondary flow rates and mass flow rates in the primary and secondary flow directions are derived. They found that maximum velocity profiles occur near the channel walls which indicates the formation of boundary layers near the walls. It has been noticed that the magnetic field decreases the temperature of the fluid while the rotation, the Hall current, the tilt angle and the Prandtl number increase it.

Hinvi et al. [7] have studied the effect of the small suction Reynolds number on the stability of the fluid flow between two horizontal fixed porous parallel plates. They showed that the instability of the disturbed flow is governed by an equation called the modified Orr-Sommerfeld equation and found that the small Reynolds number of suction/injection makes the flow more stable.

Monwanou et al. [8] studied the effects of the small injection/suction Reynolds number, the Hartmann parameter, the permeability parameter and the wave number on an electrically conductive incompressible viscous fluid flow, in porous parallel plates forming a channel. Through a suitable equation called the modified Orr-Sommerfeld equation, they found that the small injection/suction has a negligible effect on the linear temporal stability of Couette's hydromagnetic flow. They noticed that the permeability parameter (Darcy number), the Lorentz force (the Hartmann parameter) and the wave number contribute to the temporal linear stability of Couette's hydromagnetic flow.

Hatziavramidisand and $\mathrm{Ku}$ [9] developed a Chebyshev integral expansion method, based on the pseudo-spectral approximation (collocation) for the solution of boundary value problems expressed by ODEs. The method has been applied to problems arising from the linear stability analysis of two parallel shear flows, the Poiseuille plane flow and the Blasius boundary layer flow. From the application it is evident that this method, in addition to the uniform convergence and high precision characteristics of spectral methods, has the capabilities to deal directly with general type boundary conditions and is easier to perform.

Nasr and El-Hawary [10] proposed an expansion procedure using Chebyshev polynomials using the El-Gendi method, which gives more precise results than those calculated by Hatziavramidisand, from the resolution of the Orr-Sommerfeld equation for the plane Poiseuille flow and the Blasius velocity profile. This method is accomplished by starting with the Chebyshev approximation for the highest order derivative and generating approximations of the lower order derivatives by integrating the highest order derivative.

Laouer et al. [11,12] studied the hydrodynamic linear stability of the external flow on a flat plate with suction or wall blowing. The flow is assumed to be a similar to a two-dimensional laminar boundary layer flow. The average speed profiles are obtained numerically for the case of suction or blowing. The eigenvalue problem associated with the Orr-Sommerfeld stability equation is solved with great precision using the Chebyshev spectral collocation method. The digital solutions facilitate the construction of complete neutral stability curves and the determination of the stability characteristics for a wide range of permeable wall intensities from strong blowing to strong suction.

A spectral method of general mathematical framework was considered by Dongarra et al. [13] and Melenk et al. [14] for hydrodynamic stability problems. They analyzed the Orr-Sommerfeld equation provided with homogeneous boundary conditions. Reddy et al. [15] also studied the pseudospectrum method and the numerical range of this Orr-Sommerfeld operator.

Hifdi et al. [16] presented a temporal linear stability analysis of a developing channel flow. They used the Chebyshev spectral collocation method to solve the Orr-Sommerfeld equation and considered, for the average flow, an analytical solution. The results of the stability study depend essentially on the shape and the amplitude of the velocity profiles imposed at the entrance of the channel.

In the present paper, we have studied the effects of the control parameters on the temporal linear stability of a dynamic boundary layer (flow of a viscous incompressible fluid) on a horizontal fixed porous flat plate. This plate is subjected to a bottom injection speed and the thickness of the boundary layer is subjected to a suction speed. We used on the one hand the Blasius equation for the basic flow and, on the other hand, a new approach based on a derivative of a stability equation called the "modified Orr-Sommerfeld equation". This derivative of the equation is solved numerically to deal with the problem of temporal linear stability.

The paper is organized as follows: Section 2 deals with the modified Orr-Sommerfeld equation, allowing to study the temporal linear stability. Section 3 indicates the method used, that is the Chebyshev 
spectral collocation. In Section 4 the results and discussions are given and the effects of the control parameters $K p$ (permeability parameter or Darcy number) and Reo (small injection/suction Reynolds number) are analyzed. The conclusion is given in the last section.

\section{Mathematical formulation}

Basic flow

For the basic flow, the fluid is assumed to be Newtonian, incompressible and a two-dimensional laminar flow. The system of equations is described by considering the boundary layer approximation and neglecting the permeability parameter and the suction / injection rate. The basic flow velocity is simulated by solving the dimensionless Blasius equation:

$$
2 F^{\prime \prime \prime}+F^{\prime \prime} F=0,
$$

with the boundary conditions:

$$
\left\{\begin{array}{l}
\frac{d F(0)}{d y}=0 \quad \text { with } \quad F(0)=F_{0} \quad \text { at the wall }(y=0), \\
\frac{d F(\infty)}{d y}=1 \quad \text { at the border }(y \rightarrow \infty) .
\end{array}\right.
$$

\section{Linear stability}

For the study of linear stability, we established a new model of stability equation: 'Modified OrrSommerfeld equation' assuming a three-dimensional (3D) flow according to the physical model below:

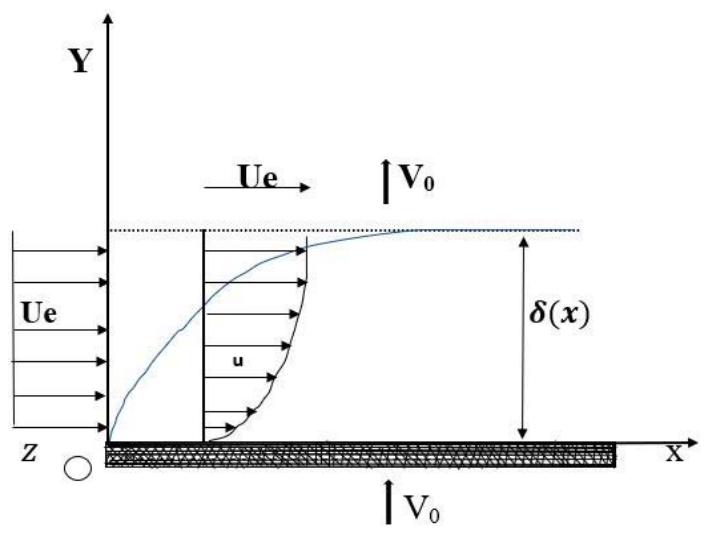

Fig1. Physical model.

The continuity and momentum equations for an incompressible Newtonian fluid are given by:

$$
\begin{aligned}
& \nabla V=0 \\
& \frac{\partial V}{\partial t}+(V \nabla) V=\frac{-1}{\rho} \nabla p+v \nabla^{2} V-\frac{\mu V}{\rho K}
\end{aligned}
$$


where $V(u, v, w)$ is the velocity.

We introduce the following additional quantities:

$$
\begin{aligned}
& t=\frac{U_{e} t}{\delta}, \quad X=\frac{x}{\delta}, \quad Y=\frac{y}{\delta}, \quad Z=\frac{z}{\delta}, \quad U=\frac{u}{U_{e}}, \\
& V=\frac{v}{V_{0}}, \quad W=\frac{w}{W_{0}}, \quad P=\frac{p}{\rho U_{e}^{2}}, \\
& R_{e}=\frac{u \delta_{x}}{v}=\frac{U_{e} \delta}{v} \text { (hydrodynamic Reynolds number), } \\
& R_{e 0}=\frac{V_{0} \delta}{v} \text { (injection/suction Reynolds number), } \\
& K_{p}=\frac{K}{\delta^{2}} \text { (permeability parameter). }
\end{aligned}
$$

Following to the axis $\mathrm{X}, \mathrm{Y}$ et $\mathrm{Z}$, equations (2.3) and (2.4) give :

$$
\begin{aligned}
& \frac{\partial U}{\partial X}+\frac{R_{e 0}}{R_{e}} \frac{\partial V}{\partial Y}+\frac{\partial W}{\partial Z}=0, \\
& \frac{\partial U}{\partial t}+U \frac{\partial U}{\partial X}+\frac{R_{e 0}}{R_{e}} V \frac{\partial U}{\partial Y}+W \frac{\partial U}{\partial Z}=\frac{-\partial P}{\partial X}+\frac{\nabla^{2} U}{R_{e}}-\frac{U}{K_{p} R_{e}}, \\
& \frac{\partial V}{\partial t}+U \frac{\partial V}{\partial X}+\frac{R_{e \theta}}{R_{e}} V \frac{\partial V}{\partial Y}+W \frac{\partial V}{\partial Z}=\frac{-R_{e}}{R_{e 0}} \frac{\partial P}{\partial Y}+\frac{\nabla^{2} V}{R_{e}}-\frac{V}{K_{p} R_{e}}, \\
& \frac{\partial W}{\partial t}+U \frac{\partial W}{\partial X}+\frac{R_{e 0}}{R_{e}} V \frac{\partial W}{\partial Y}+W \frac{\partial W}{\partial Z}=\frac{-\partial P}{\partial Z}+\frac{\nabla^{2} W}{R_{e}}-\frac{W}{K_{p} R_{e}} .
\end{aligned}
$$

For the analysis of stability, one superposes on the basic flow $U$ parallel, a perturbation represented by $u^{\prime}(x, y, z, t), v^{\prime}(x, y, z, t), w^{\prime}(x, y, z, t), p^{\prime}(x, y, z, t)$ with:

$$
\begin{aligned}
& U=U(y)+u^{\prime} ; \quad V=V_{0}+v^{\prime} ; \quad W=W_{0}+w^{\prime} ; \\
& P=P(x)+p^{\prime}, \quad V_{0}=1 \quad \text { and } \quad W_{0}=0, \\
& \frac{u}{U} \ll 1, \quad \frac{v}{V} \ll 1, \quad \frac{w}{W} \ll 1 .
\end{aligned}
$$


Equations (2.6)-(2.8) give:

$$
\begin{aligned}
& \frac{\partial u^{\prime}}{\partial t}+U \frac{\partial u^{\prime}}{\partial x}+\frac{R_{e 0}}{R_{e}} \frac{\partial u^{\prime}}{\partial y}+\frac{R_{e 0}}{R_{e}} v^{\prime} \frac{\partial u^{\prime}}{\partial y}=\frac{-\partial p^{\prime}}{\partial x}+\frac{\nabla^{2} u^{\prime}}{R_{e}}-\frac{u^{\prime}}{K_{p} R_{e}} \\
& \frac{\partial v^{\prime}}{\partial t}+U \frac{\partial v^{\prime}}{\partial x}+\frac{R_{e 0}}{R_{e}} \frac{\partial v^{\prime}}{\partial y}=\frac{-R_{e}}{R_{e 0}} \frac{\partial p^{\prime}}{\partial y}+\frac{\nabla^{2} v^{\prime}}{R_{e}}-\frac{v^{\prime}}{K_{p} R_{e}}, \\
& \frac{\partial w^{\prime}}{\partial t}+U \frac{\partial w^{\prime}}{\partial x}+\frac{R_{e 0}}{R_{e}} \frac{\partial w^{\prime}}{\partial y}=\frac{-\partial p^{\prime}}{\partial z}+\frac{\nabla^{2} w^{\prime}}{R_{e}}-\frac{w^{\prime}}{K_{p} R_{e}} .
\end{aligned}
$$

The perturbations have the form $f^{\prime}(x ; y ; z ; t)=\dot{f}(y) \operatorname{expi}(k x+\xi z-\alpha t)$, where $f^{\prime}$ represents each of $u^{\prime}, v^{\prime}, w^{\prime} \vee p^{\prime}$ and $\dot{f}$ the amplitude; $k=\beta x=\beta \cos (\theta)$ and $\xi=\beta z=\beta \sin (\theta)$ are numbers of waves, respectively, following the directions of the axes $x$ and $z ; \alpha=k c$ is the frequency of the wave; $i^{2}=-1$, $\theta=(\beta x ; \beta), c=c r+i c i$ is the complex speed, $k \wedge \xi$ are real numbers due to temporal analysis. We have then:

$$
\begin{aligned}
& -i k c \dot{u}+i k U \dot{u}+\frac{R_{e 0}}{R_{e}} \dot{v} U^{\prime}+\frac{R_{e 0}}{R_{e}} \frac{\partial \dot{u}}{\partial y}=-i k \dot{p}-\frac{\beta^{2}}{R_{e}}+\frac{1}{R_{e}} \frac{\partial^{2} \dot{u}}{\partial y^{2}}-\frac{\dot{u}}{K_{p} R_{e}}, \\
& -i k c \dot{v}+i k U \dot{v}+\frac{R_{e 0}}{R_{e}} \frac{\partial \dot{v}}{\partial y}=\frac{-R_{e}}{R_{e 0}} \frac{\partial \dot{p}}{\partial y}-\frac{\beta^{2}}{R_{e}}+\frac{1}{R_{e}} \frac{\partial^{2} \dot{v}}{\partial y^{2}}-\frac{\dot{v}}{K_{p} R_{e}}, \\
& -i k c \dot{w}+i k U \dot{w}+\frac{R_{e 0}}{R_{e}} \frac{\partial \dot{w}}{\partial y}=-i \xi \dot{p}-\frac{\beta^{2}}{R_{e}}+\frac{1}{R_{e}} \frac{\partial^{2} \dot{w}}{\partial y^{2}}-\frac{\dot{w}}{K_{p} R_{e}} .
\end{aligned}
$$

From Eqs (2.12) and (2.14), one obtains:

$$
\begin{aligned}
& -i k \frac{R_{e 0}}{R_{e}}(U-c) D^{2} \dot{v}-\frac{R_{e 0}^{2}}{R_{e}^{2}} D^{3} \dot{v}+i k \frac{R_{e 0}}{R_{e}} \dot{v} U^{\prime \prime}= \\
& =\beta^{2} D \dot{p}+\beta^{2} \frac{R_{e 0}}{R_{e}^{2}} D^{2} \dot{v}-\frac{R_{e 0}}{R_{e}^{2}} D^{4} \dot{v}+\frac{R_{e 0}}{K_{p} R_{e}^{2}} D^{2} \dot{v} \\
& i(k \dot{u}+\xi \dot{w})=\frac{-R_{e 0}}{R_{e}} \frac{\partial \dot{v}}{\partial y}=\frac{-R_{e 0}}{R_{e}} D \dot{v}
\end{aligned}
$$

where D is the Chebyshev's spectral differentiation matrix. From Eq.(2.13), we derive the pressure gradient which we replace in the relation Eq.(2.15):

$$
i k\left[(U-c)\left(D^{2}-\beta^{2}\right)-U^{\prime \prime}\right] \dot{v}=\frac{1}{R_{e}}\left(D^{2}-\beta^{2}\right)^{2} \dot{v}-\left(\frac{R_{e 0}}{R_{e}} D+\frac{1}{K_{p} R_{e}}\right)\left(D^{2}-\beta^{2}\right) \dot{v}
$$


Equation (2.17) can be written as:

$$
\begin{aligned}
& {\left[-i k U\left(D^{2}-\beta^{2}\right)+i k U^{\prime \prime}+\frac{1}{R_{e}}\left(D^{2}-\beta^{2}\right)^{2}-\left(\frac{R_{e 0}}{R_{e}} D+\frac{1}{K_{p} R_{e}}\right)\left(D^{2}-\beta^{2}\right)\right] \dot{v}=} \\
& =c\left[-i k\left(D^{2}-\beta^{2}\right)\right] \dot{v},
\end{aligned}
$$

with the boundary conditions:

$$
\dot{v}(0)=\dot{v}\left(y_{\infty}\right)=\dot{v}^{\prime}(0)=\dot{v}^{\prime}\left(y_{\infty}\right) .
$$

Equation (2.18) is that of modified stability, here called the Orr-Sommerfeld equation modified by the permeability parameter $K_{p}$ and the small Reynolds number $R_{e 0}$. It can be written in the form of an eigenvalues equation:

$$
H \varphi=c G \varphi
$$

where $c$ and $\varphi=v$ are respectively eigenvalues and eigenfunctions; $H$ and $G$ are matrix differential operators:

$$
\begin{aligned}
& H=-i k U\left(D^{2}-\beta^{2}\right)+i k U^{\prime \prime}+\frac{1}{R_{e}}\left(D^{2}-\beta^{2}\right)^{2}-\left(\frac{R_{e 0}}{R_{e}} D+\frac{1}{K_{p} R_{e}}\right)\left(D^{2}-\beta^{2}\right), \\
& G=-i k\left(D^{2}-\beta^{2}\right) .
\end{aligned}
$$

\section{Numerical method}

\section{Mean flow solution}

For the numerical resolution of the base flow velocity profile $U$, its first derivative $U$ ' and its second derivative U", we used the fourth-order Runge-Kuta method. Thus, we have written the third order equation Eq.(2.1), with the boundary conditions Eq.(2.2), in three first order equations that we have treated in the Fortran environment.

\section{Spectral method solution}

To solve our new model (modified Orr-Sommerfeld equation) and make this work numerically reliable, we used Chebyshev's spectral collocation method which is better suited for linear stability studies. This method is well detailed in $[17,19]$.

So, to leave the domain of Chebyshev $-1 \leq z \leq 1$ for that of the boundary layer $0 \leq y \leq y_{\infty}$, we made the following transformations:

$$
\begin{aligned}
& y=y_{\infty} \frac{1+z}{2}, \\
& \hat{D}=\frac{2}{y_{\infty}} D,
\end{aligned}
$$


with $\hat{D}$, the new differentiation matrix.

$$
\left\{\begin{array}{l}
y=0 \quad \text { for } \quad z=-1 \\
y=y_{\infty} \quad \text { for } \quad z=1 .
\end{array}\right.
$$

So the modified Orr-Sommerfeld equation Eq.(2.18) can be rewritten as a new eigenvalue equation:

$$
\hat{H}\left(k, \beta, R_{e}\right) \hat{\varphi}=c \hat{G}\left(k, \beta, R_{e}\right) \hat{\varphi},
$$

with $\hat{\varphi}$ the discrete eigen function; $\hat{H}$ and $\hat{G}$, matrix differential operators:

$$
\begin{aligned}
& \hat{H}=-i k U\left(\hat{D}^{2}-I \beta^{2}\right)+i k I U^{\prime \prime}+\frac{1}{R_{e}}\left(\hat{D}^{2}-I \beta^{2}\right)^{2}-\left(\frac{R_{e 0}}{R_{e}} \hat{D}+\frac{I}{K_{p} R_{e}}\right)\left(\hat{D}^{2}-I \beta^{2}\right), \\
& \hat{G}=-i k\left(\hat{D}^{2}-I \beta^{2}\right)
\end{aligned}
$$

where $I$ is the identity matrix of order $M \times M$.

The problem of generalized eigenvalues Eq.(3.4) linked to the boundary conditions Eq.(2.19) in the boundary layer is solved using the QZ algorithm. This algorithm makes it possible to calculate the neutral stability curve, for a given average speed profile $U(y)$, by finding the values of $\mathrm{k}$ and of Re at which $\mathrm{c}$ is real; from where $c i=0$.

\section{Results and discussion}

Basic flow velocity profile

Figure 2 represents the variations of the dimensionless velocity and their first and second derivatives of the base flow as a function of the dimensionless coordinate $y$.

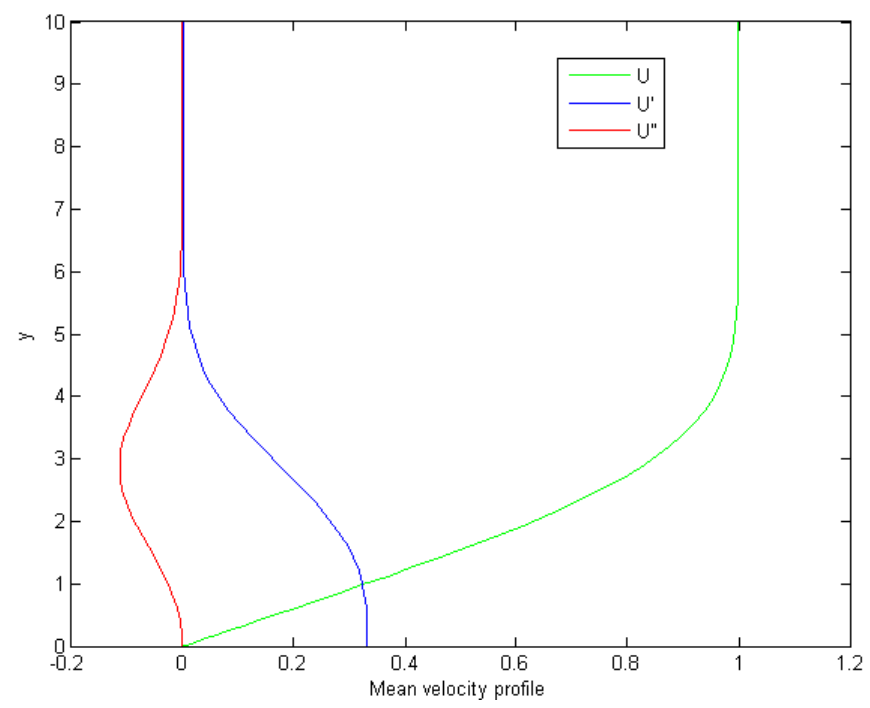

Fig.2. Speed profile. 


\section{Stability analysis results}

Figure 3 represents the stability curves of the flow for some values of the imaginary part (ci) of the wave propagation speed. These stability curves are presented in the form of contour plots in the $(\Re ; k)$ plane. This figure shows that the interior of the neutral marginal curve $(c i=0)$ is unstable $(c i>0)$ and its exterior is stable $(c i<0)$. This is done for $K_{p}=7199$ and Reo=0 (Fig.3(a)), for $K_{p}=7199$ and $R_{e 0}=2$ (Fig.3(b)) and for $K_{p}=0.04$ and $R_{e 0}=0$ (Fig.3(c)) with $F_{0}=0$ in each case .

Figure 4 also represents the curves of marginal stability of the flow for some values of the permeability parameter $K_{p}$ with $R_{e 0}=0$ and $F_{0}=0$. Each curve in this figure separates the stable area from the unstable area of the flow.

Note that the increase in the permeability parameter causes a widening of the instability zone. This increase causes a gradual decrease in the Reynolds number $\mathfrak{R}$ and a gradual increase in the $k$ wave numbers, which makes the flow more unstable.

We also notice that there is a threshold $\left(K_{p}=7199\right)$ of the values of $K_{p}$, from which the zone of instability becomes invariant, i.e. the Reynolds and wave numbers are constant (Tab.1.).

Table 1. Critical flow parameters with the effect of $K_{p}$, for $R_{e 0}=0$ and $F_{0}=0$.

\begin{tabular}{|l|c|c|c|c|c|c|c|c|}
\hline$K_{p}$ & 0.04 & 0.1 & 0.3 & 1 & 7198 & 7199 & 10000 & 1000000000 \\
\hline$R_{e c}$ & 7590.1 & 2454.1 & 1053.9 & 665.9 & 531.3 & 531.2 & 531.2 & 531.2 \\
\hline$k_{c}$ & 0.1525 & 0.2135 & 0.2570 & 0.2870 & 0.3025 & 0.3030 & 0.3030 & 0.3030 \\
\hline
\end{tabular}

Table 2. Comparison of $R_{e c}$ with or without $K p$ effect, for $R_{e 0}=0$ and $F_{0}=0$.

\begin{tabular}{|c|c|l|l|c|c|}
\hline & $\begin{array}{l}\text { present } \\
\text { results }\end{array}$ & $\begin{array}{l}\text { present } \\
\text { results }\end{array}$ & $\begin{array}{l}\text { Laour } \text { et al. } \\
{[11,12]}\end{array}$ & Boiko et al. [21] & $\begin{array}{l}\text { Venkatasobbaiah and } \\
\text { Sengupta [20] }\end{array}$ \\
\hline$\frac{1}{K_{p}}$ & $\begin{array}{c}\text { not } \\
\text { neglected }\end{array}$ & neglected & neglected & neglected & Neglected \\
\hline$R e c$ & 531.2 & 520.06 & 519.4 & 520 & 519.018 \\
\hline
\end{tabular}

Thus, $\forall K_{p} \geq 7199$, the domain of instability is constant and the critical Reynolds and corresponding wave numbers are $R_{e c}=531.2$ and $k c=0.303$, respectively.

It is therefore retained here that the permeability parameter (Darcy number) plays the role of injection (increase in instability) in the hydrodynamic boundary layer on a porous flat plate.

Moreover, when we compared this critical value $R_{e c}=531.2$ to those of the literature, we noted that there is a difference (Tab.2.); which is quite normal. This difference is due to the fact that the coefficient $\frac{1}{K_{p}}$ of Eq.(2.18) cannot be zero $\left(\frac{1}{K_{p}}>0, \forall K_{p} \in 0 ;+\infty\right)$. But when we assumed in Eq.(2.18) that $\frac{1}{K_{p}}$ is negligible $\left(\frac{1}{K_{p}} \approx 0\right)$ with $R_{e 0}=0$ ( then: $-\left(\frac{R_{e 0}}{R_{e}} D+\frac{1}{K_{p} R_{e}}\right)\left(D^{2}-\beta^{2}\right) \approx 0$ ), we found $R_{e c}=520.06$ which is in good agreement with the results of the literature where the effect of the Darcy number is not considered (Tab.2.). 
Figure 5 illustrates the effect of the small Reynolds number on the temporal linear stability which is highlighted. Thus, for $K_{p}=7199$ and $F_{0}=0$, the increase in the small Reynolds number $\left(R_{e o}\right)$ leads to a slight reduction in the zone of instability (Fig.5(a)); this reduction is clearly observed in the zoomed part (Fig.5(b)).

Similarly, for $K_{p}=0.04$ and $F_{0}=0$ (Fig.6.), the zone of instability is contracted by the effect of the small Reynolds number (Fig.6(a)), which is quite clear in Fig.6(b). It is therefore maintained here that the small Reynolds number plays the role of suction (reduction of instability) in the hydrodynamic boundary layer.

Figure 7 represents the spectrum of eigenvalues for $K_{p}=1, R_{e 0}=0$ (Fig.7(a)); for $K_{p}=50, R_{e 0}=0$ (Fig.7(b)) and for $K_{p}=1, R_{e 0}=0.5$ (Fig.7(c)); with $F_{0}=0, R_{e}=1000$ and $k=0.2$ in each case.

a)

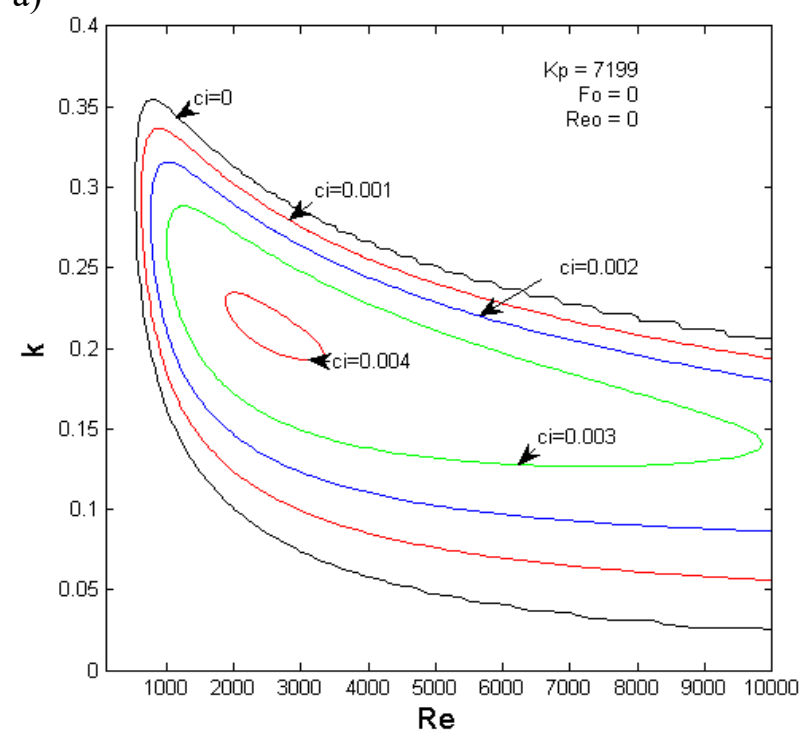

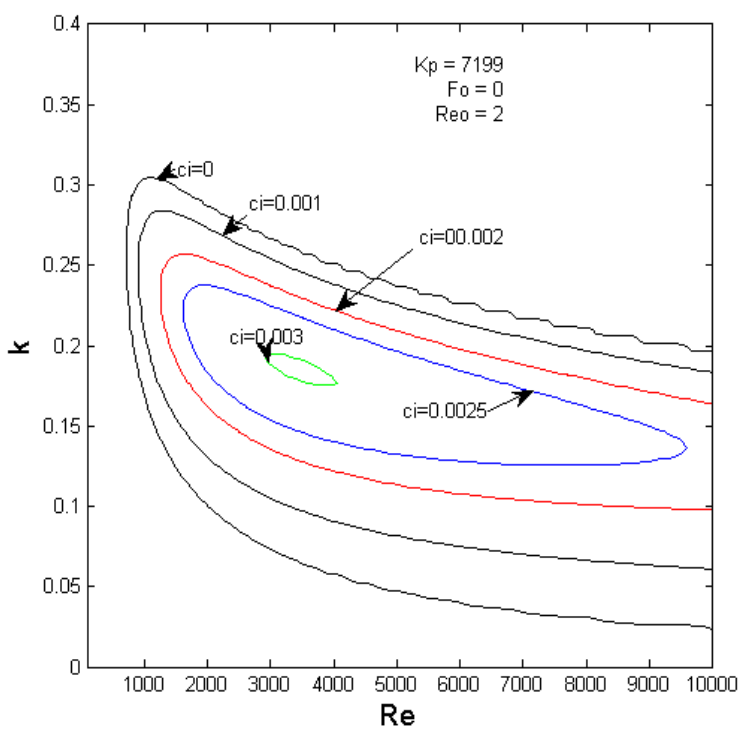

$\mathrm{Re}$

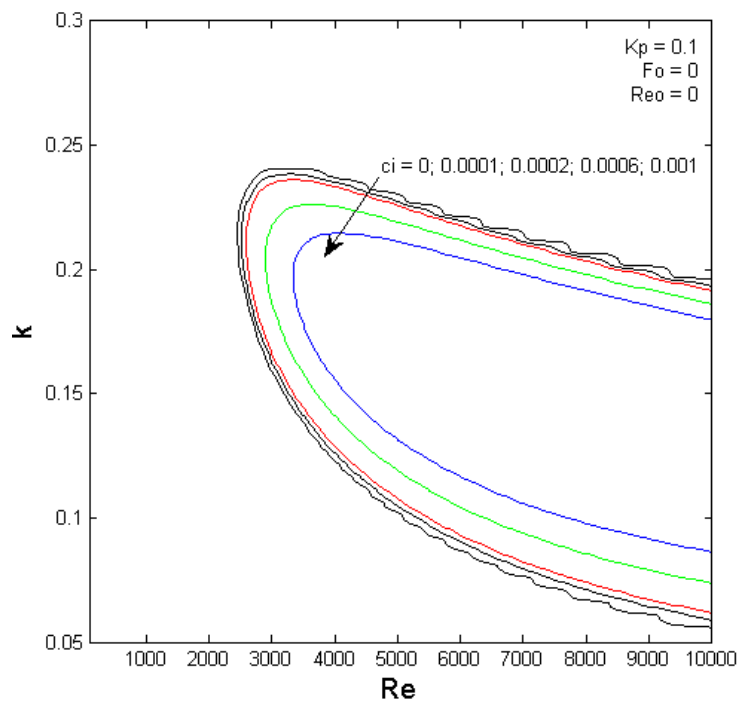

Fig.3. Stability curves for different values of $c i$ : a) $K_{p}=7199$ and $R_{e 0}=0$; b) $K_{p}=7199$ and $R_{e 0}=2$, c) $K_{p}=0.1$ and $R_{e 0}=0$. 


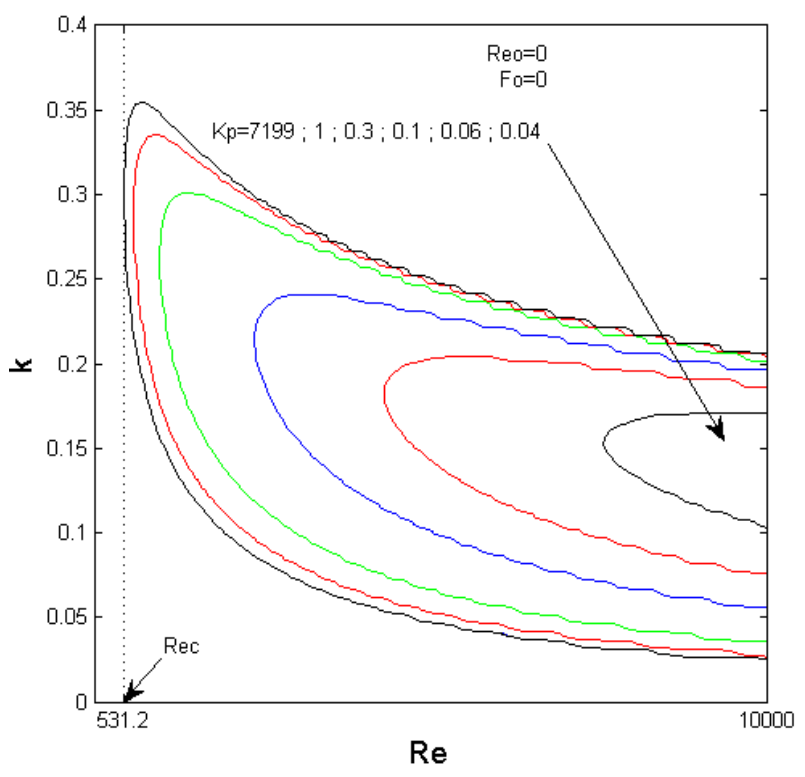

Fig.4. Marginal stability curves for $R_{e 0}=0, F_{0}=0$ and varying $K_{p}$.

Here, the maximum values of $c i$ for the three spectra ((Fig.7(a): $c=0.569234+0.00339 i)$; (Fig.7(b): $c=9.50+0.43610 i)$; (Fig.7(c): $c=0: 572231+0: 000997 i)$ ) confirmed the results according to which the permeability parameter $K_{p}$ contributes to the widening of the instability zone of the dynamic laminar boundary layer and the small Reynolds $R_{e 0}$ number, its reduction.

a)

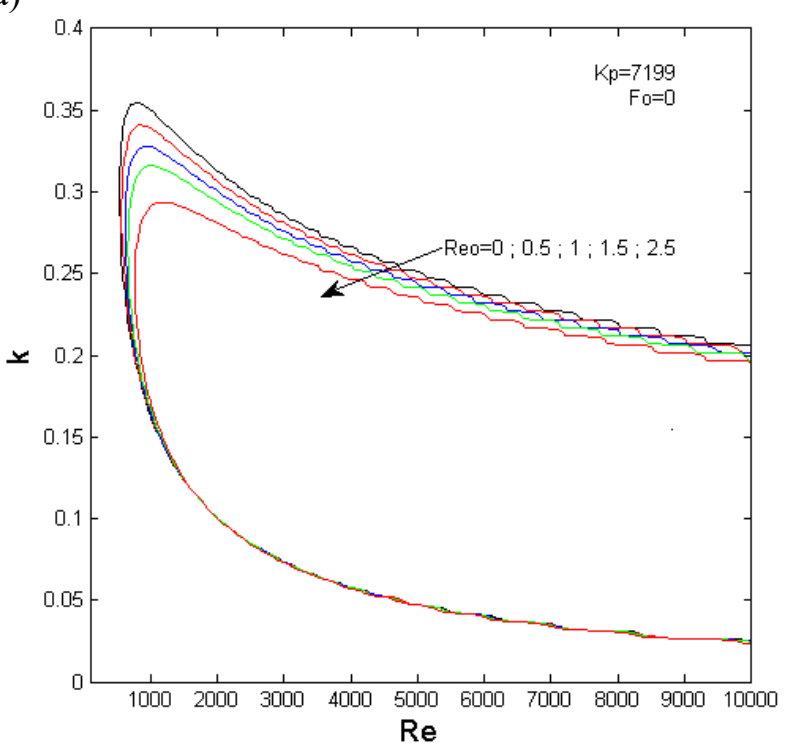

b)

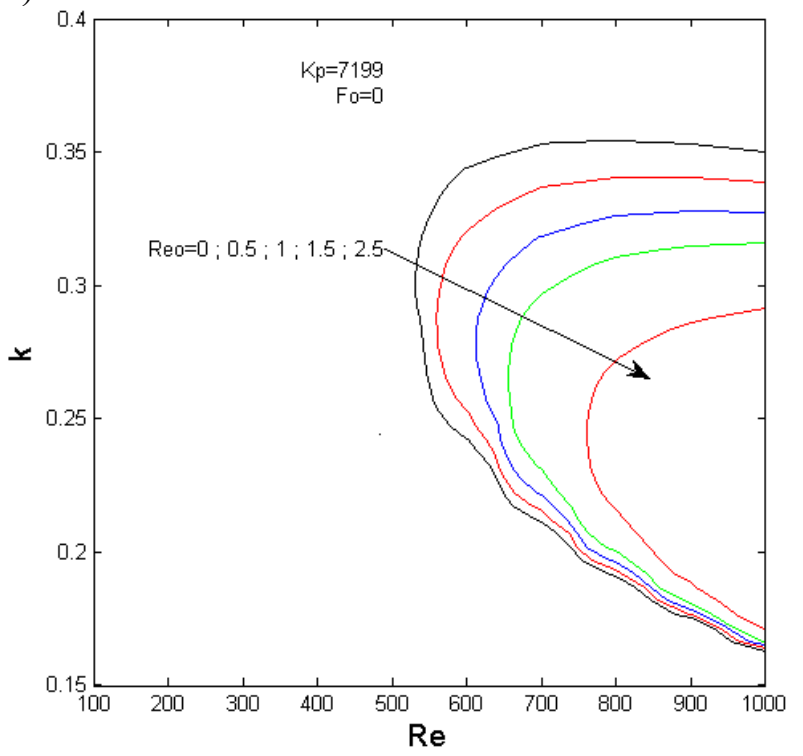

Fig.5. Marginal stability curves for $K_{p}=7199, F_{0}=0$ and varying $R_{e 0}$. 
a)

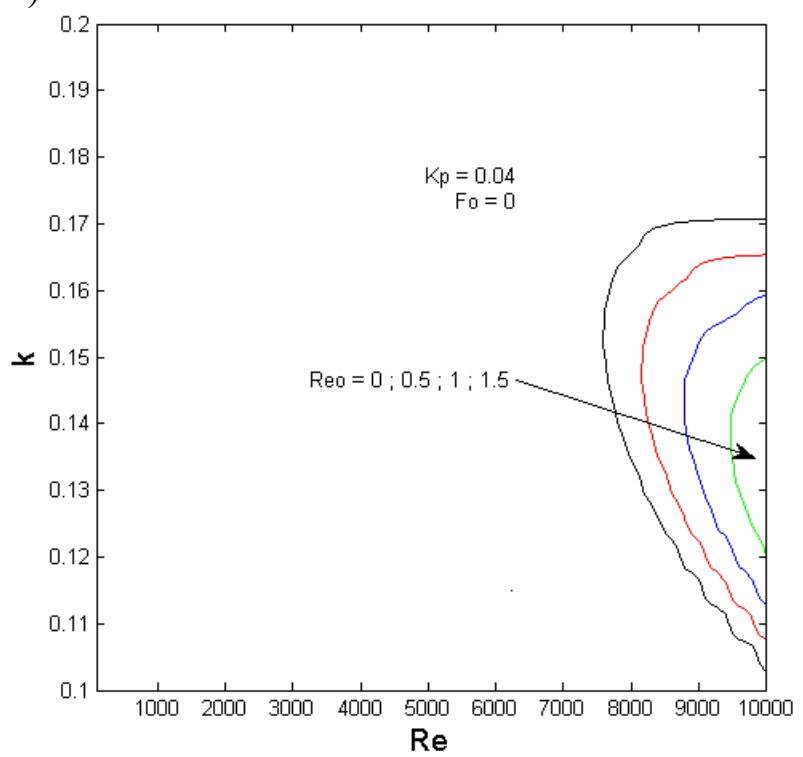

b)

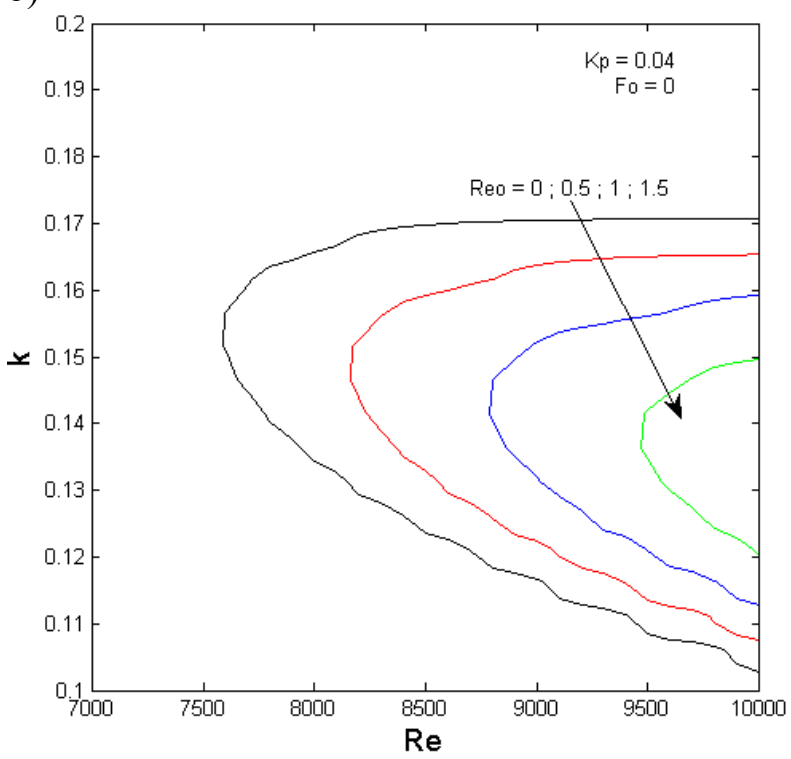

Fig.6. Marginal stability curves for $K_{p}=0.04, F_{0}=0$ and varying $R_{e 0}$

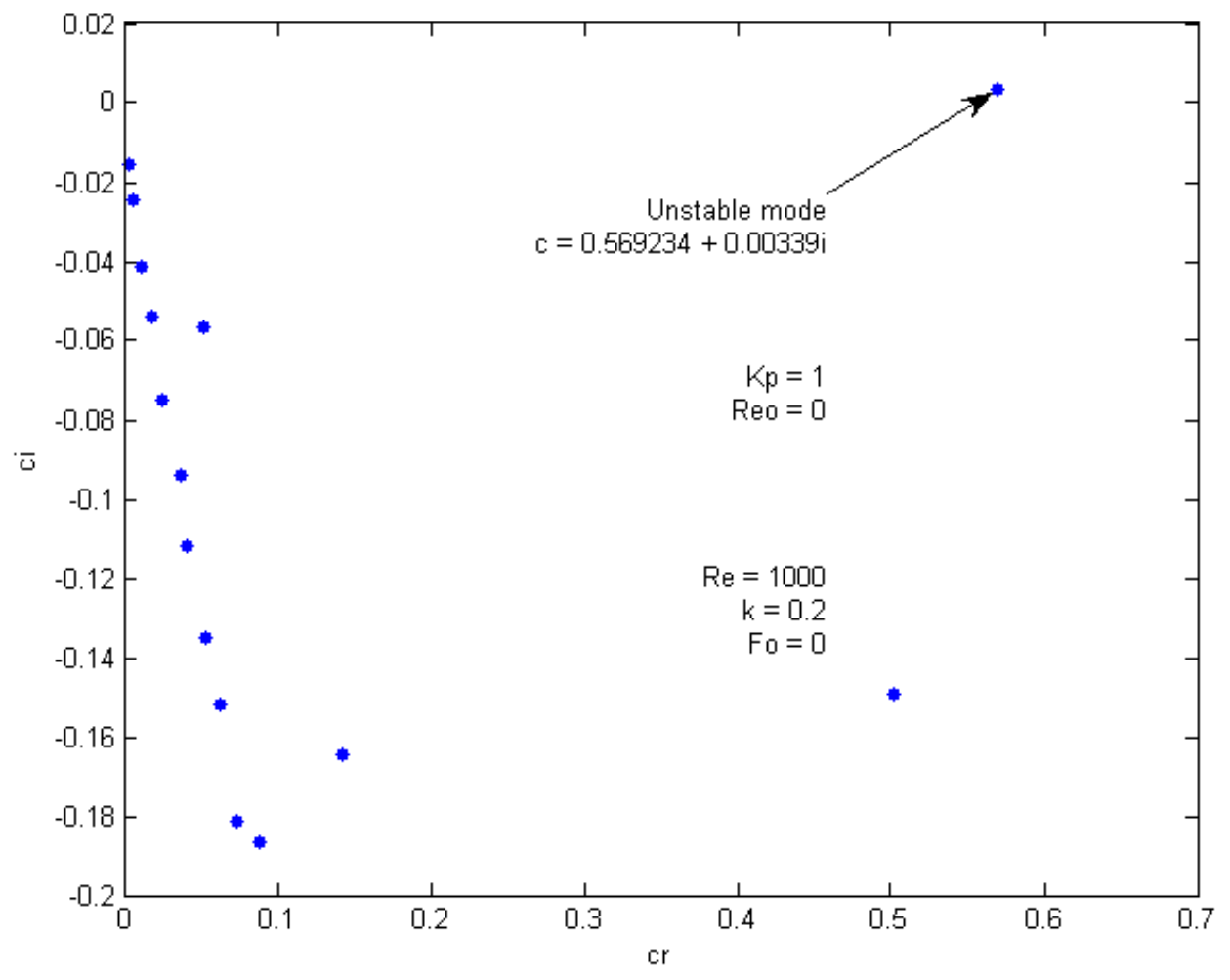

Fig.7(a). Eigenvalue spectra for $K_{p}=1$ and $R_{e 0}=0$. 


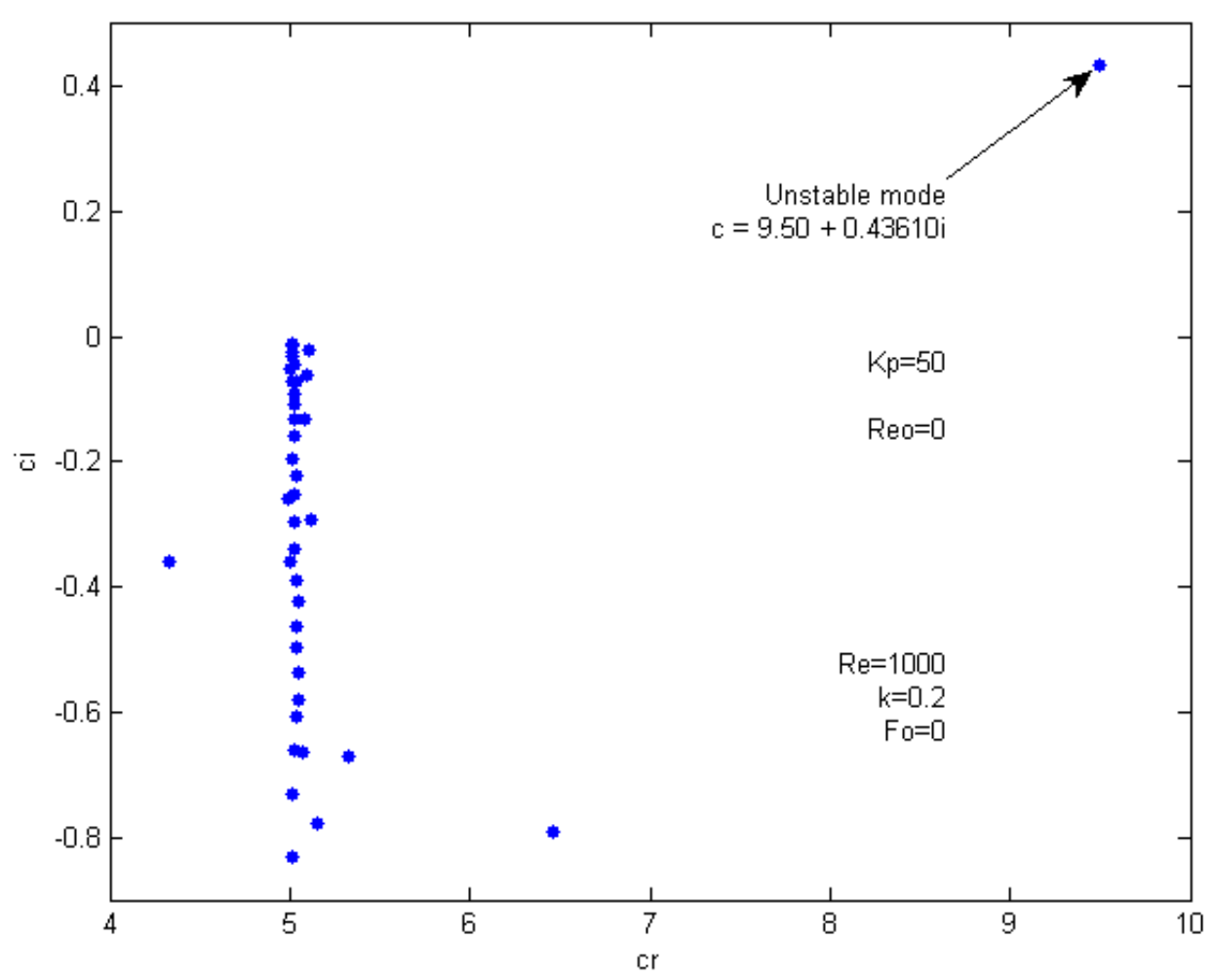

Fig.7(b). Eigenvalue spectra $K_{p}=50$ and $R_{e 0}=0$.

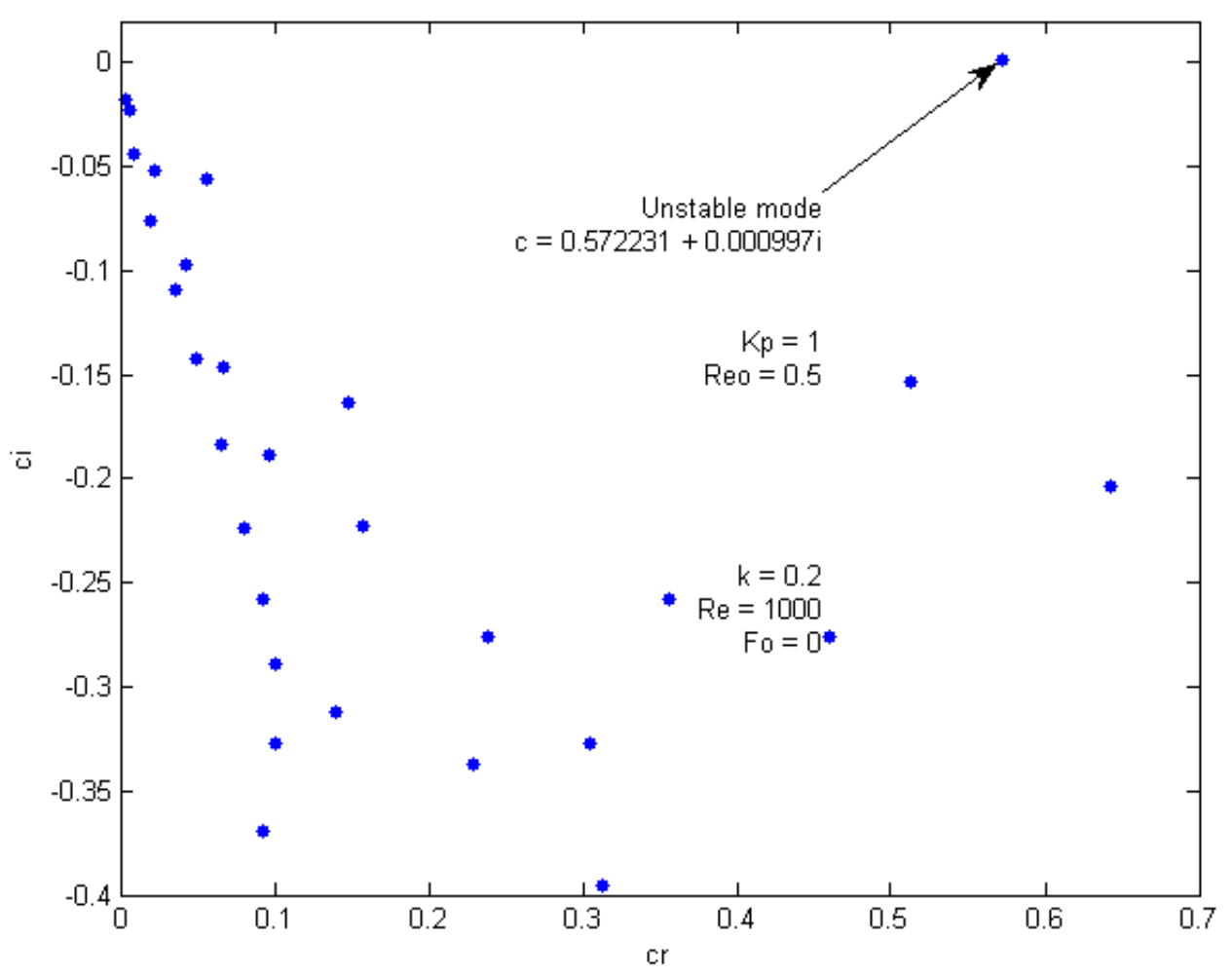

Fig.7(c). Eigenvalue spectra $K_{p}=1$ and $R_{e 0}=0.5$. 


\section{Conclusion}

In this work we have studied the linear temporal stability of a dynamic laminar boundary layer on a horizontal permeable flat plate received an injection/aspiration speed. We assumed the laminar and twodimensional base flow and obtained the base velocity profiles numerically by the fourth-order Runge-Kutta method applied to the Blasius equation. The formulation of the stability problem leads to the Orr-Sommerfeld equation modified by the permeability parameter (Darcy number) and the small injection/suction Reynolds number. This equation is solved numerically by Chebyshev's spectral collocation method. This method allowed us to obtain the diagrams of marginal stability, the critical Reynolds numbers and the spectra of the eigenvalues for different values of the permeability parameter and the small imposed Reynolds number. The numerical solutions allow us to claim that the increase in the permeability parameter makes the dynamic boundary layer more unstable while the increase in the small Reynolds number makes it stable.

\section{Acknowlegments}

The authors thank The World Academy of Sciences (TWAS) for financial support through Research Grant Agreement $N^{\circ}$ 20-307 RG/PHYS/AF/AC_G-FR3240314170. The authors would like to thank very much the anonymous referees whose useful criticisms, comments and suggestions have helped improve the content and the quality of the paper.

\section{Nomenclature}

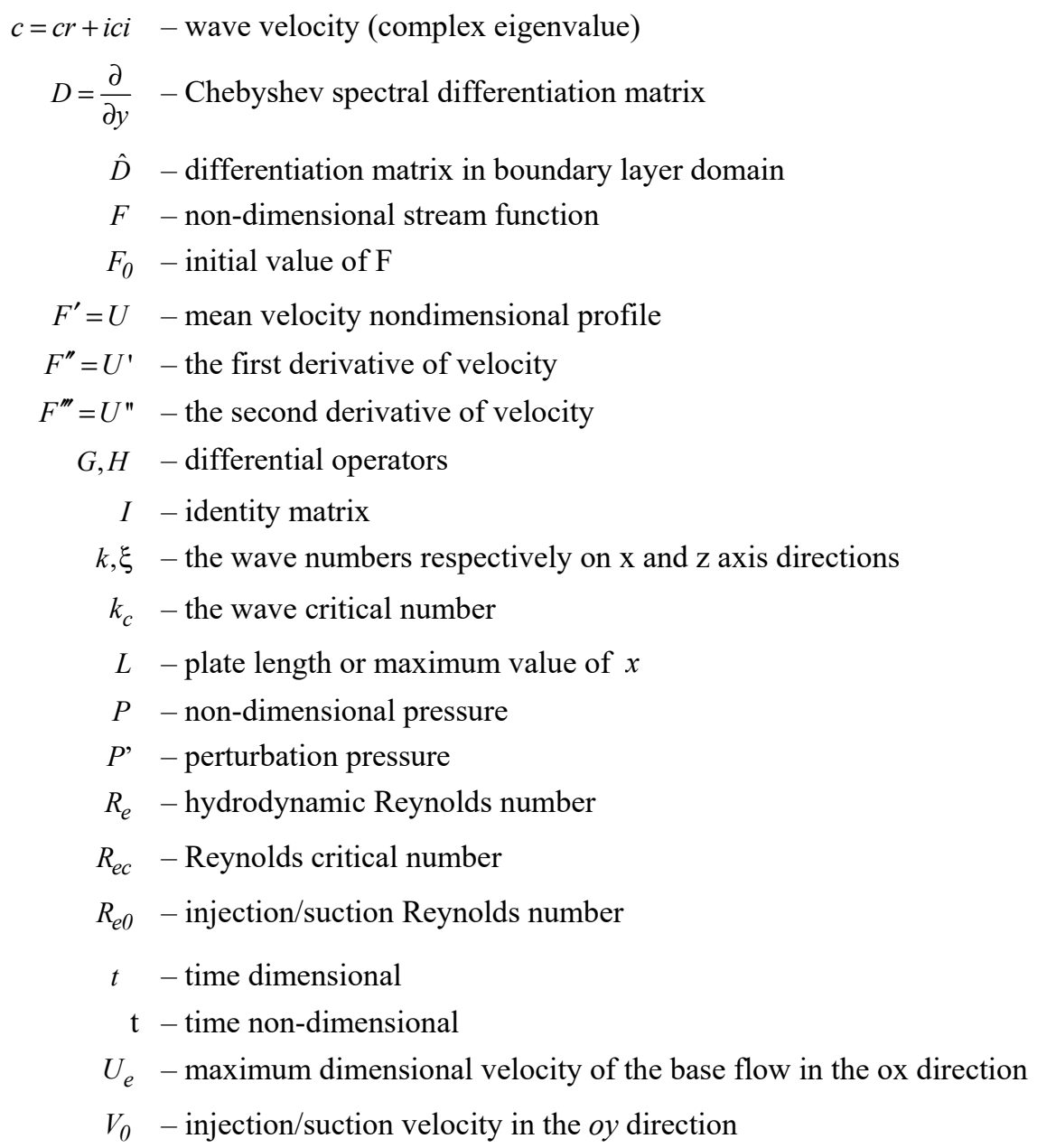




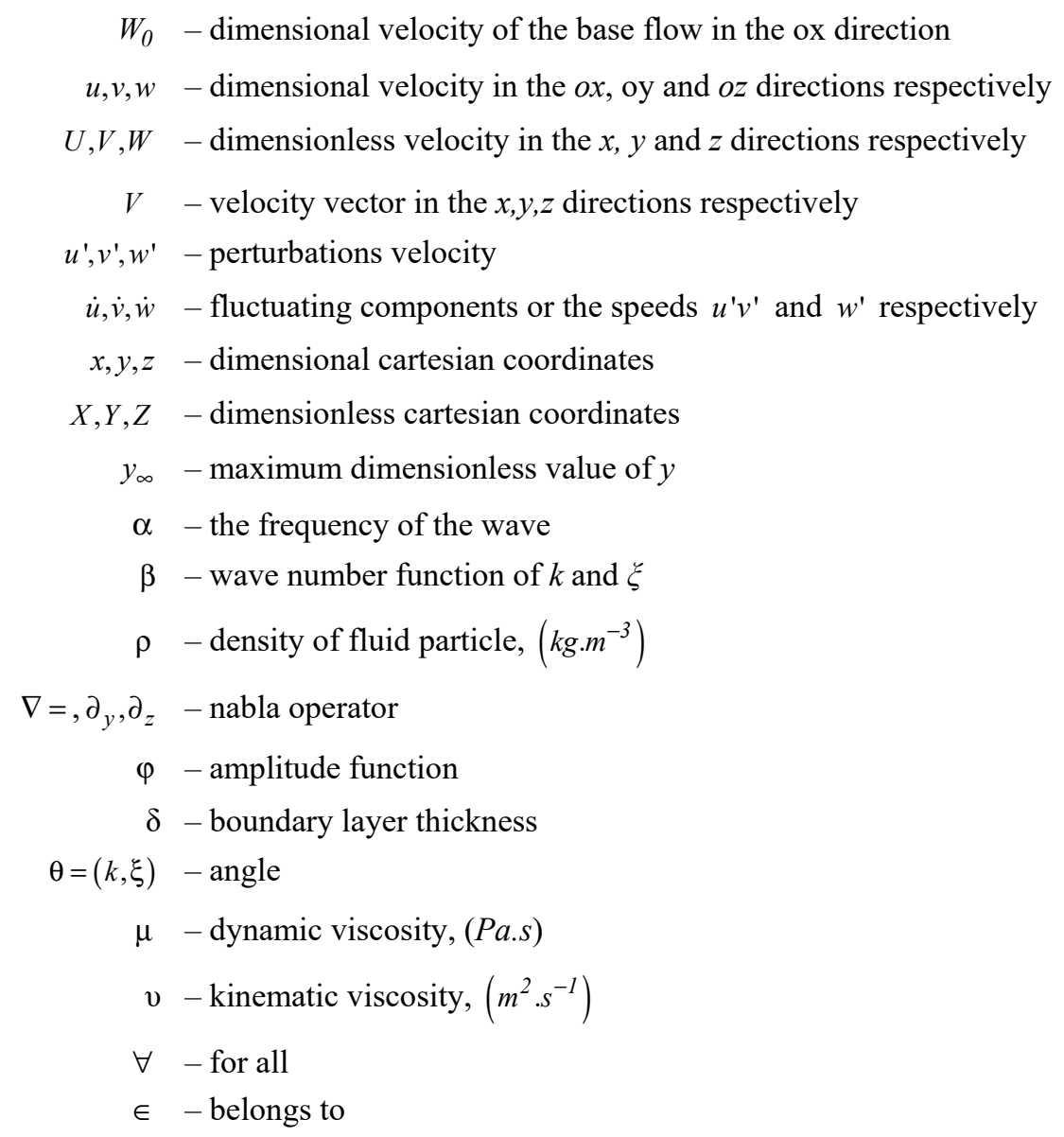

\section{References}

[1] Wartemann V., Wagnerb A., Kuhn M., Eggers T. and Hannemann K. (2015): Passive hypersonic boundary layer transition control using an ultrasonically absorptive coating with random microstructure: Computational analysis based on the ultrasonic absorption properties of carbon-carbon.- Procedia IUTAM, vol.14, pp.413-422.

[2] Wang Y., Li S. and Yang X. (2016): Numerical investigation of the passive control of cavity flow oscillations by a dimpled non-smooth surface.- Applied Acoustics, vol.111, pp.16-24.

[3] Simonn B., Nemitz T., Rohlfing J., Fischer F., Mayer D. and Grundmann S. (2015): Active flow control of laminar boundary layers for variable flow conditions.- International Journal of Heat and Fluid Flow, vol.56, pp.344-354.

[4] Svorcan J., Stupar S., Trivkovic S., Petrasinovic N. And Ivanov T. (2014): Active boundary layer control in linear cascades using CFD and artificial neural networks.- Aerospace Science and Technology, vol.39, pp.243-249.

[5] Ming-Liang, Jian-Zhong L. and Fu-Tang X. (2007): On the hydrodynamic stability of a particle-laden flow in growing flat plate boundary layer.- J. Univ. Sci A: App. Ph. Eng., vol.8, No.2, pp.275-284, DOI:0.1631/jzus.2007.A0275.

[6] Seth G.S., Raj Nandkeolyar and Ansari Md.S. (2010): Hartmann flow in a rotating system in the presence of inclined magnetic field with Hall effects.- Tamkang Journal of Science and Engineering, vol.13, No.3, pp.243-252.

[7] Hinvi L.A., Monwanou V.A. and Chabi Orou J.B. (2014): Linear stability analysis of fluid flow between two parallel porous stationary plates with small suction and injection.- The African Review of Physics, vol.9, pp.115-121.

[8] Monwanou A.V., Hinvi A.L., Miwadinou H.C. and Chabi Orou J.B. (2017): A new approach for the stability analysis in hydromagnetic Couette flow.- Journal of Applied Mathematics and Physics, vol.5, pp.1503-1514. https://doi.org/10.4236/jamp.2017.57123.

[9] Hatziavramidisand D. and Ku H.C. (1985): An integral Chebyshev Expansion Method for boundary-value problems of O.D.E. type.- Comp. Maths. with Appls., vol.11, No.6, pp.581-586. DOI: 10.1016/0898-1221(85)90040-9. 
[10] Nasr H. and El-Hawary H.M. (1991): A Chebyshev method for the solution of boundary value problems.International Journal of Computer Mathematics, vol.40, No.3-4, pp.251-258, DOI:10.1080/00207169108804019.

[11] Laouer A., Mezaache E. and Laouar S. (2016): Study of the effect of parietal suction and blowing on the stability of laminar external flow.- International Journal of Heat and Technology, vol.34, No.2, pp.302-310.

[12] Laouer A., Mezaache E. and Laouar S. (2016): Influence of surface mass transfer on the stability offorced convection flow over an horizontal flat plate. -Computational Thermal Sciences, Begell House, vol.8, No.4, pp.355-369.

[13] Dongarra J.J., Straughan B. and Walker D.W. (1996): Chebyshev tau-QZ algorithm methods for calculating spectra of hydrodynamic stability problems.- Appl. Numer. Math., vol.22, pp.399-434.

[14] Melenk J.M., Kirkner N.P. and Schwab V. (2000): Spectral Galerkin discretization for hydrodynamic stability problems.- Comput., vol.65, pp.97-118.

[15] Reddy S. C., Schmid P.J. and Henningson D. (1993): Pseudospectra of Orr-Sommerfeld operator.- SIAM J. Appl. Math., vol.53, pp.15-47.

[16] Hifdi A., Touhami M.O. and Naciri J.K. (2004): Channel entrance flow and its linear stability.- J. Stat. Mech.: Theory Exp., vol.2004, p.06003.

[17] Peyret R. (2002): Spectral Methods for Incompressible Viscous Flow.- Applied Mathematical Sciences, SpringerVerlag, New York.

[18] Zebib A. (1984): A Chebyshev method for the solution of boundary value problems.- J. Comput. Phys., vol.53, pp.443-455.

[19] Boyd J.P. (2001): Chebyshev and Fourier Spectral Methods.- Dover Publications, Mineola, NY.

[20] Venkatasobbaiah K. and Sengupta T.K. (2009): Mixed convection flow past a vertical plate: Stability analysis and its direct simulation.- Int. J. Therm. Sci., vol.48, pp.461-474.

[21] Boiko A.V., Dovgal A.V., Grek G.R. and Kozlov V.V. (2012): Physics of Transitional Shear Flows.- Instability and Laminar Turbulent Transition in Incompressible Near-Wall Shear Layers.- Springer, New York.

[22] Motsa S.S., Marewo G.T., Sibanda P. and Shateyi S. (2011): An improved spectral homotopy analysis method for solving boundary layer problems.- Boundary Value Problems, Springer Open Journal, Article No.3.

Received: June 4, 2021

Revised: September 9, 2021 